\title{
miR-204 Inhibits Epithelial to Mesenchymal Transition by Targeting Slug in Intrahepatic Cholangiocarcinoma Cells
}

\author{
Ying-he Qiu ${ }^{a, c}$ Yong-peng Wei ${ }^{b, c}$ Ning-jia Shen ${ }^{a, c}$ Zhou-chong Wang ${ }^{b, c}$ Tong Kan ${ }^{a}$ \\ Wen-long Yu ${ }^{a}$ Bin Yia Yong-jie Zhang ${ }^{a}$ \\ aThe Second Department of Biliary Tract Surgery, Eastern Hepatobiliary Surgery Hospital, The Second

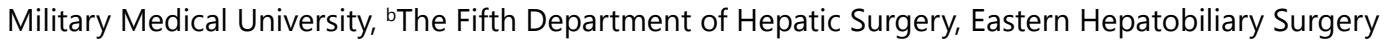 \\ Hospital, The Second Military Medical University, Shanghai, China; 'These authors contributed equally \\ to this work
}

\section{Key Words}

miR-204 • Slug • Intrahepatic cholangiocarcinoma • EMT

\begin{abstract}
Background/Aims: MicroRNAs (miRNAs) play critical roles during carcinogenesis and cancer progression. Down-regulation of miR-204 has been frequently observed in various cancers. In this study, we investigated the roles and mechanisms of miR-204 in human intrahepatic cholangiocarcinoma (ICC). Methods: The relative expression of miR-204 in ICC tissues and cell lines was monitored by qRT-PCR. Effects of miR-204 were studied in human ICC cell lines HuH28 and HuCCT1, and cells were analyzed for proliferation, migration and invasion. Expression levels of miR-204 target gene Slug and EMT markers (E-cadherin and vimentin) in ICC cell lines and tissues were measured by qRT-PCR, western blotting and immunofluorescence. Results: miR-204 was frequently downregulated in human ICC, and the low-level expression of miR-204 was significantly associated with lymph node metastasis. Overexpression of miR-204 dramatically suppressed ICC cell migration and invasion, as well as the epithelialmesenchymal transition process (EMT). Slug was identified as a direct target of miR-204, and its downregulation by miR-204 in HuH28 cells reversed EMT, as shown by the increased expression of the epithelial marker E-cadherin and decreased expression of the mesenchymal marker vimentin. Conclusion: These findings suggest that miR-204 plays negative roles in the invasive and/or metastatic potential of ICC, and that its suppressive effects are mediated by repressing Slug expression.
\end{abstract}




\section{Introduction}

Intrahepatic cholangiocarcinoma (ICC) is the second most common type of primary liver cancer (PLC), and both its incidence rate and mortality are increasing drastically in recent years $[1,2]$. Like many other solid tumors, metastases are the main cause of death from ICC. In order to metastasize, tumor cells must pass through a multistep process in which local invasion is considered to be an initial, essential step leading to the formation of a secondary tumor nodule [3]. Recent studies have shown that aberrant activation of the embryonic morphogenetic program, termed the epithelial-mesenchymal transition (EMT), is frequently implicated in tumor cell invasion [4]. EMT is a complex process that enables cancer cells to dissolve their cell-cell junctions, acquire migratory properties, and become motile mesenchymal cells $[5,6]$. This process of EMT is associated with the downregulation of the epithelial marker E-cadherin and the upregulation of mesenchymal marker vimentin [7]. Several transcription factors are also known to play a central role in the activation of EMT, such as SNAI1 (Snail), SNAI2 (Slug), ZEB1 and ZEB2, all of which directly repress E-cadherin gene transcription $[8,9]$.

MicroRNAs (miRNAs) are a class of endogenous noncoding RNAs of 20-22 nucleotides. Processed mature miRNA can interact with the 3'-untranslated region (UTR) of target messenger RNA (mRNA) causing degradation and/or translation repression [10,11]. Through down-regulating the target gene expression, miRNAs play important roles in regulating almost every biological process, including cell proliferation, apoptosis, differentiation, and migration $[12,13]$. Meanwhile, a large body of evidence indicates that abnormal expression of miRNAs correlates with various cancers including ICC. Several miRNAs have been reported to be dysregulated in ICC, such as miR-21, miR-124 and miR-214 [14-16], which act as tumor suppressors or oncogenes in the progression of ICC. In addition, miRNAs function as crucial modulators for EMT. The miR-200 family and miR-205 have been identified as EMT-suppressive miRNAs directly targeting ZEB1, SIP1, or NCAM1, thereby reducing the aggressiveness of cancer cells $[17,18]$.

Recently, a previous profile study of miRNA expression has documented a downregulation of a series of miRNAs, including miR-204, in ICC tissues [19]. miR-204 has been shown to be down-regulated and serve as a potential tumor suppressor in several distinct cancer types including endometrial cancer, malignant peripheral nerve sheath tumors, gastric cancer, glioma, and hepatocellular carcinoma [20-25]. However, the role of miR-204 in ICC progression and the molecular mechanisms by which miR-204 exerts its functions remain largely unknown.

In this study, we found that miR-204 was downregulated in ICC tissues and cell lines. Overexpression of miR-204 in ICC cells suppressed cell migration and invasion. More importantly, we provide novel evidence that miR-204 overexpression inhibited EMT by directly targeting Slug. Our findings provide a clearer understanding of the role of miR-204 in ICC metastasis and suggest a potential application of miR-204 in cancer treatment.

\section{Materials and Methods}

\section{Tissue samples and cell lines}

Human ICC tissues were obtained from patients who had undergone ICC surgery at Eastern Hepatobiliary Surgery Hospital, Second Military Medicine University (Shanghai, China). Clinicopathological characteristics of the patients are presented in Table 1. The tissues were snap-frozen in liquid nitrogen and stored at $-80^{\circ} \mathrm{C}$ until use. Written informed consent was obtained from each patient and this study was approved by the Ethics Committee of Human Experimentation of the Second Military Medical University. The human ICC cell lines ICC-9810, RBE were obtained from Shanghai Cell Bank of Chinese Academy of Sciences (Shanghai, China); HuH28 and HuCCT1 were from Cell Bank, RIKEN BioResource Center, Tsukuba, Japan. All cells were cultured in RPMI 1640 medium (Invitrogen, Carlsbad, CA, USA) containing 


\begin{tabular}{|c|c|c|}
\hline Cellular Physiology & Cell Physiol Biochem 2013;32:1331-1341 & \\
\hline and Biochemistry & $\begin{array}{l}\text { DOI: } 10.1159 / 000354531 \\
\text { Publisnea oninne: November 22, } 2013\end{array}$ & $\begin{array}{l}\text { O } 2013 \text { S. Karger AG, Basel } \\
\text { www.karger.com/cpb }\end{array}$ \\
\hline
\end{tabular}

$10 \%$ fetal bovine serum, $100 \mathrm{U} / \mathrm{mL}$ penicillin, and $100 \mathrm{mg} /$ $\mathrm{mL}$ streptomycin (Invitrogen). Cells were incubated $37^{\circ} \mathrm{C}$ in a humidified chamber containing $5 \% \mathrm{CO}_{2}$.

RNA isolation and quantitative real-time PCR ( $q R T-P C R$ )

Total RNA was extracted from tissues or cells using TRIzol reagent (Invitrogen) following the manufacturer's instructions. miRNAs were isolated using a miRNeasy Mini Kit (Qiagen, Valencia, CA, USA). The PCR primers for miR-204 and RNU6B were obtained from RiBoBio (Guangzhou, China). The primers for Slug were 5'-TGT TGC AGT GAG GGC AAG AA-3' (forward) and 5'-GAC CCT GGT TGC TTC AAG GA-3' (reverse). qRT-PCR reactions were performed on the ABI PRISM 7900HT System (Applied Biosystems, Foster City, CA, USA) using SYBR Premix Ex Taq II (TaKaRa, Dalian, China). The RNU6B RNA or $\beta$-actin levels were used as normalization controls, and the relative expression levels were calculated using the $2^{-\Delta \Delta \mathrm{ct}}$ method. All reactions were performed in triplicate.

Lentivirus infection and oligonucleotide transfection

The pre-miR-204 sequence and Slug coding sequence were obtained from Origene (Rockville, MD, USA). The sequences were cloned into the pGCSIL-GFP lentiviral vector. The production, purification, and titration of lentivirus was performed as previously described [26]. HuH28 and HuCCT1 cells were infected with the recombinant lentivirus-transducing units in the presence of $8 \mu \mathrm{g} / \mathrm{mL}$ polybrene (Sigma-Aldrich, St. Louis, MO, USA). Empty lentiviral vector was used as a control. Anti-miR-204 and negative control were designed and synthesized by Qiagen. HuCCT1 cells were transfected with antimiR-204 or anti-NC using Lipofectamine 2000 (Invitrogen). Cells were collected $48 \mathrm{~h}$ after transfection.

Plasmid construction and luciferase assays

The 3'UTR fragment of Slug (NM_003068) was amplified by PCR using the following primers: 5'-AAT GCG GCC GCA GTC TGT AAT AGG ATT TCC CAT AGG-3' (forward) and 5'-CTA
Table 1. Clinicopathological characteristics of ICC Patients $(n=30)$

\begin{tabular}{|c|c|c|}
\hline Characteristic & $\begin{array}{l}\text { Case } \\
\text { Number }\end{array}$ & $\%$ \\
\hline \multicolumn{3}{|l|}{ Sex } \\
\hline Male & 24 & 80.0 \\
\hline Female & 6 & 20.0 \\
\hline \multicolumn{3}{|l|}{ Age (years) } \\
\hline$\leq 60$ & 10 & 33.3 \\
\hline$>60$ & 20 & 66.7 \\
\hline \multicolumn{3}{|l|}{ HBsAg } \\
\hline Negative & 16 & 53.3 \\
\hline Positive & 14 & 46.6 \\
\hline \multicolumn{3}{|l|}{ Cirrhosis } \\
\hline Yes & 5 & 16.7 \\
\hline No & 25 & 83.3 \\
\hline \multicolumn{3}{|l|}{ Tumor size } \\
\hline$\leq 5 \mathrm{~cm}$ & 8 & 26.7 \\
\hline$>5 \mathrm{~cm}$ & 22 & 73.3 \\
\hline \multicolumn{3}{|l|}{ TNM stage } \\
\hline I/ II & 18 & 60.0 \\
\hline III/IV & 12 & 40.0 \\
\hline
\end{tabular}

GAG CTC CGC CAG GAA TGT TCA AAG CTA ATC T-3' (reverse). To mutate the binding site of miR-204, its complementary sequence in the 3'UTR of Slug (AAAGGGA) was replaced by AAGACGA. The PCR products were digested using NotI and $\mathrm{XhoI}$ and inserted into the psiCHECK2 vector (Promega, Madison, WI, USA). miR-204 expressing or control cells were seeded into 24-well plates and cotransfected with the wild-type (WT) or mutated Slug 3'UTR reporter plasmids and pRL-TK using Lipofectamine 2000 reagent (Invitrogen). Luciferase assays were performed $48 \mathrm{~h}$ after transfection using the Dual Luciferase Reporter Assay System (Promega). All experiments were performed in triplicate.

\section{Cell proliferation assay}

Cell proliferation assay was performed using Cell Counting Kit-8 (Dojindo, Kumamoto, Japan) according to the manufacturer's instructions. Briefly, infected cells $\left(2 \times 10^{3}\right)$ were seeded onto each well of 96-well plates in a final volume of $100 \mu \mathrm{L}$, and cultured for $24,48,72$, and $96 \mathrm{~h}$. CCK-8 solution $(10 \mu \mathrm{L})$ was added into each well, and the cells were incubated for $4 \mathrm{~h}$ at $37^{\circ} \mathrm{C}$. After incubating, the absorbance at $450 \mathrm{~nm}$ was measured to calculate the number of viable cells. Each experiment was performed 3 times independently. 
Cell migration and invasion assays

A 24-well transwell plate (8- $\mu$ m pore size, Corning, New York, USA) was used to examine the migratory and invasive ability of ICC cells. For migration assays, cells $\left(2 \times 10^{4}\right)$ were resuspended in $100 \mu \mathrm{l}$ of serumfree RPMI 1640 medium and plated into the upper chamber of the insert. For invasion assays, chamber inserts were coated with $150 \mu \mathrm{g}$ of Matrigel and dried overnight under sterile conditions. Then, $1 \times 10^{5}$ cells were added into the upper chamber. The lower chamber was filled with RPMI 1640 with 10\% fetal bovine serum. After incubation at $37{ }^{\circ} \mathrm{C}$ for $24 \mathrm{~h}$, the cells remaining on the upper surface of the membrane were removed, whereas the cells that had invaded through the membrane were fixed in $100 \%$ methanol, stained with $0.2 \%$ crystal violet, imaged, and counted under a microscope (Olympus, Tokyo, Japan). All experiments were performed in triplicate.

\section{Western blotting}

Whole cell protein lysates were separated on 10\% SDS-PAGE and electrotransferred to a polyvinylidene difluoride membrane (Millipore, Billerica, MA, USA). After blocking in 5\% nonfat dried milk solution for 2 $\mathrm{h}$, the membrane-bound proteins were probed with primary antibodies overnight at $4^{\circ} \mathrm{C}$. The membranes were washed and then incubated with HRP-conjugated secondary antibodies. Signals were visualized with enhanced chemiluminescence reagents (Millipore). The following primary antibodies were used: E-cadherin, vimentin, Snail, Slug, ZEB1, ZEB2 (Cell Signaling, Beverly, MA, USA), and $\beta$-actin (Santa Cruz Biotechnology Inc., Santa Cruz, CA, USA).

\section{Immunofluorescence}

Cells were plated onto glass coverslips in 24-well plates and fixed for $30 \mathrm{~min}$ in $4 \%$ paraformaldehyde. The cells were permeabilized with $0.04 \%$ Triton X-100 for $10 \mathrm{~min}$, and then probed with a primary antibody against Slug (Cell Signaling) for $1 \mathrm{~h}$ at $37^{\circ} \mathrm{C}$, followed by 3 washings with PBS and incubation with antirabbit-Alexa 594-conjugated antibodies (Invitrogen) for $30 \mathrm{~min}$ at room temperature. To detect nuclei, cells were counterstained with 4',6-diamidino-2-phenylindole , and images were taken on a fluorescence microscope (Olympus).

\section{Statistical analysis}

Statistical analysis was performed with SPSS 12.0. Data are expressed as the mean \pm SD from at least three independent experiments. The difference between groups was analyzed using Student $t$-test when comparing only two groups or one-way analysis of variance when comparing more than two groups. $P$ values of $<0.05$ was considered statistically significant.

\section{Results}

Down-regulation of miR-204 is associated with metastasis of ICC

In this study, the expression levels of miR-204 were first measured by quantitative realtime PCR (qRT-PCR) in 20 pairs of ICC and adjacent normal tissues. As shown in Fig. 1A, miR204 expression was significantly reduced in ICC tissues compared to their matched normal tissues. Furthermore, in comparison to non-metastatic ICC tissues, miR-204 levels were significantly lower in metastatic ICC tissues (Fig. 1A). Consistent with these observations, the expression of miR-204 was significantly decreased in four ICC cell lines compared with normal tissues (Fig. 1B). These results indicate that reduced expression of miR-204 might play a critical role in ICC progression.

miR-204 inhibits the migration and invasion of ICC cells

To explore the potential biological function of miR-204 in ICC progression, we established stable-expression of miR-204 HuH28 cells by lentivirus infection, as HuH28 cells showed the lowest expression level of miR-204 in ICC cell lines (Fig. 1C). Successful re-expression of miR-204 was verified by qRT-PCR (Fig. 2A). We found that overexpression of miR-204 had no effect on proliferation of HuH28 cells (Fig. 2B). Intriguingly, miR-204 overexpression significantly suppressed the migration and invasion of HuH28 cells (Fig. 2C 


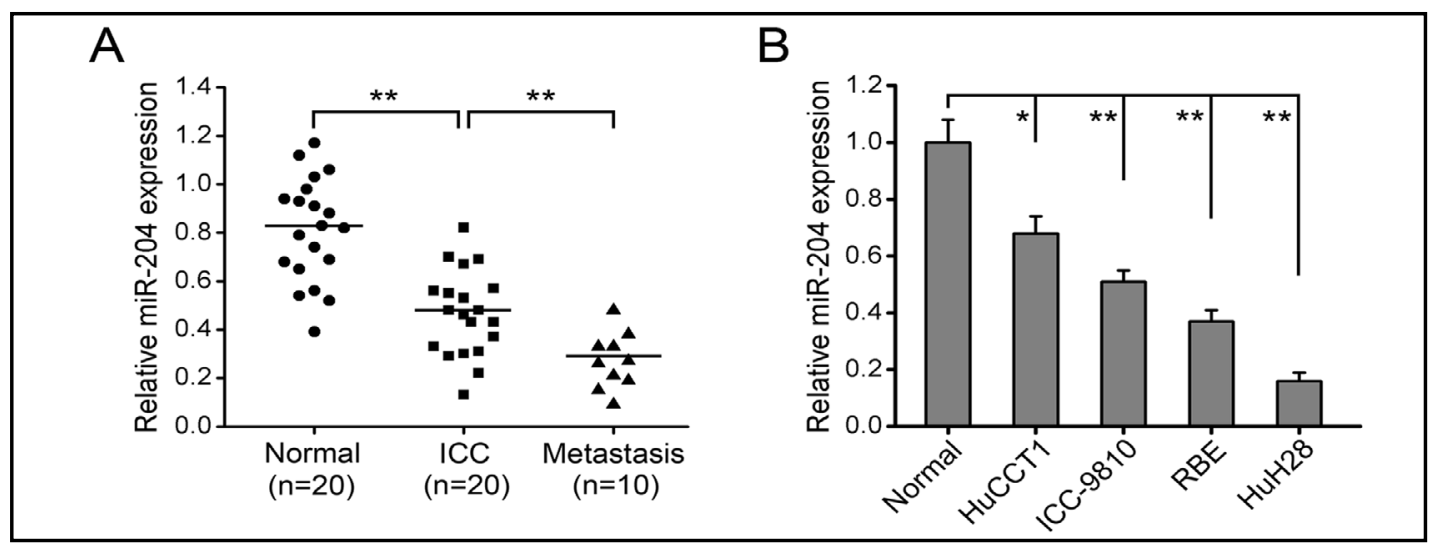

Fig. 1. Expression of miR-204 in human ICC tissues and cell lines. (A) Relative expression of miR-204 in matched normal tissues, primary ICC tissues and lymph node metastatic tissues. (B) Relative expression of miR-204 in human ICC cell lines HuCCT1, ICC-9810, RBE and HuH28. The expression of miR-204 was quantified by qRT-PCR and normalized to RNU6B. ${ }^{*} P<0.05$, ${ }^{* *} P<0.01$.

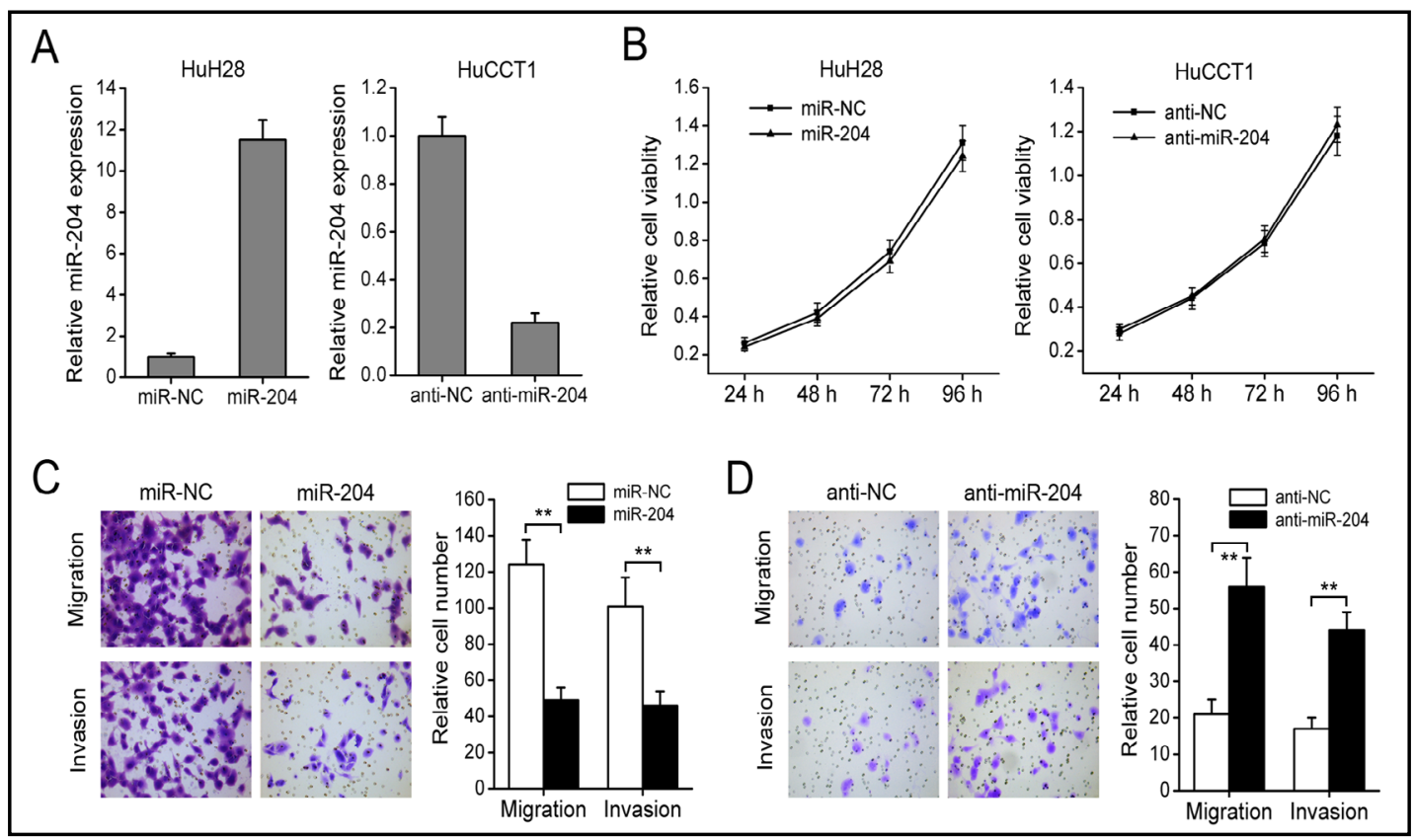

Fig. 2. miR-204 suppresses ICC cell migration and invasion in vitro. (A) HuH28 cells were infected with miR204 or miR-NC lentivirus; HuCCT1 cells were transfected with anti-miR-204 or anti-NC. The expression of miR-140 was analyzed by qRT-PCR. RNU6B was used as an internal control. (B) cell viability assay (CCK-8). (C, D) Transwell migration and invasion assays. ${ }^{* *} P<0.01$.

and D). In contrast, we transiently transfected miR-204 inhibitor into HuCCT1 cells, which had relatively higher endogenous miR-204 expression, and successful inhibition of miR-204 expression was confirmed by qRT-PCR (Fig. 2A). As expected, miR-204 depletion did not change cell proliferation, but significantly increased cell migration and invasion in HuCCT1 cells (Fig. 2B and D). Altogether, these results demonstrate that miR-204 significantly inhibits the migration and invasion of ICC cells in vitro.

miR-204 suppresses EMT in ICC cells

In HuH28 cells, we observed that upregulation of miR-204 resulted in cell morphological changes from an elongated, fibroblast-like, mesenchymal phenotype to an epithelial 


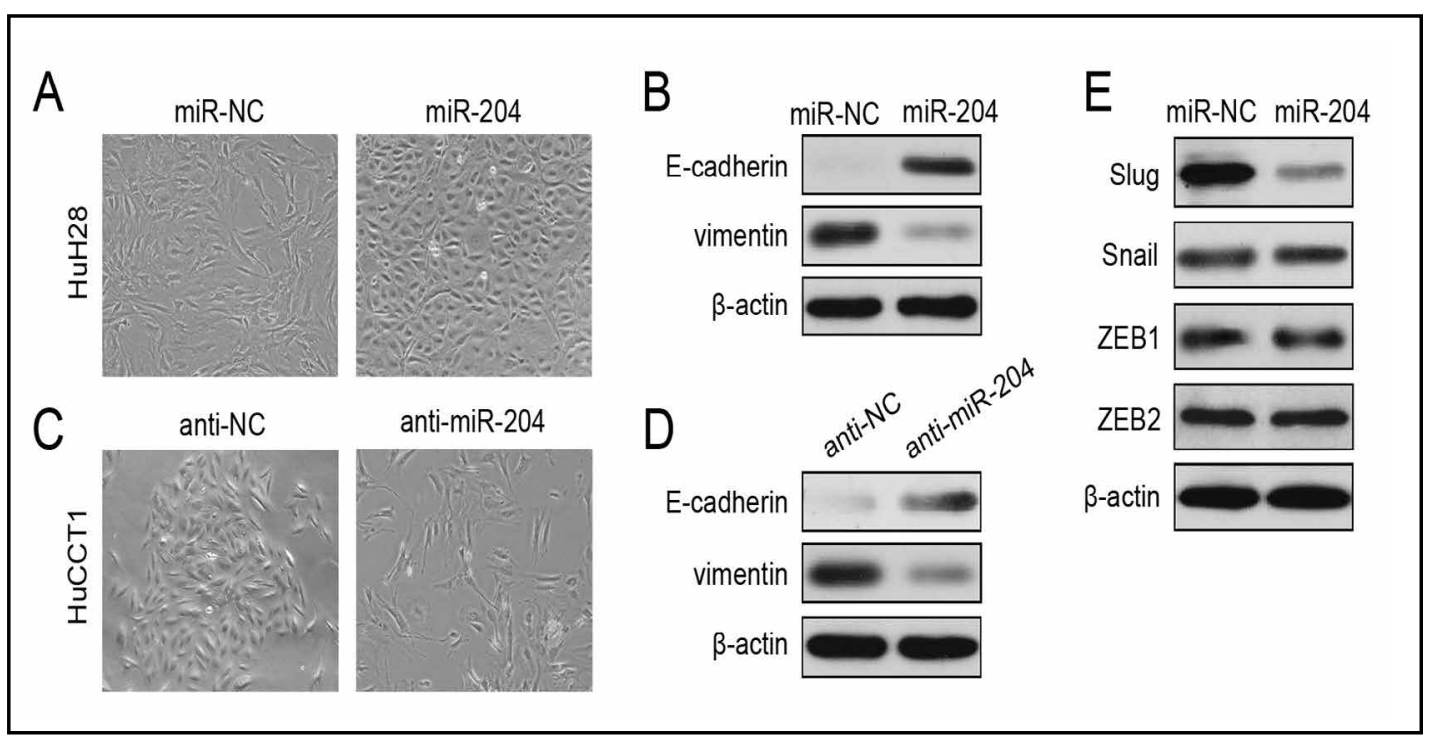

Fig. 3. miR-204 suppresses EMT in ICC cells. (A) Morphological changes of HuH28 cells infected with miR204 or miR-NC lentivirus. (B) Western blotting analysis of E-cadherin and vimentin in HuH28 cells infected with miR-204 or miR-NC lentivirus. (C) Morphological changes of HuCCT1 cells transfected with antimiR-204 or anti-NC. (D) Western blotting analysis of E-cadherin and vimentin in HuCCT1 cells transfected with anti-miR-204 or anti-NC. (E) Western blotting analysis of Slug, Snail, ZEB1 and ZEB2 in HuH28 cells infected with miR-204 or miR-NC lentivirus.

cobblestone-like phenotype (Fig. 3A). These morphological changes were also associated with reduced expression of vimentin and increased expression of E-cadherin (Fig. 3B). Conversely, HuCCT1 cells with miR-204 depletion showed morphological changes consistent with those of EMT (Fig. 3C). The epithelial marker E-cadherin showed robust upregulation, while the expression of mesenchymal marker vimentin decreased (Fig. 3D). These results suggest that miR-204 can partially repress EMT in ICC cells. Furthermore, we evaluated the effects of miR-204 overexpression on the expression of Snail, Slug, ZEB1 and ZEB2, which are direct transcriptional repressors of E-cadherin and potent EMT inducers, in HuH28 cells. Western blotting analysis showed that miR-204 overexpression significantly reduced Slug expression, whereas the expression of other factors had no significant changes (Fig. 3E), suggesting that Slug may play an important role in the miR-204-induced cell effects.

\section{Slug is a direct target of miR-204}

To explore the mechanisms by which miR-204 inhibits EMT in ICC, we used two publicly available databases, miRanda and TargetScan, to identify potential targets of miR204, especially those that are relevant to EMT. The transcription factor Slug was identified as a candidate target of miR-204, because the complementary sequence of miR-204 was identified in its 3'UTR (Fig. 4A), and most importantly, its expression is reduced with upregulation of miR-204 (Fig. 3B). To test whether Slug is a direct target of miR-204, the wild-type or mutant miR-204 target sequences of the Slug 3'UTR was cloned into the region immediately downstream of the Renilla luciferase gene. Luciferase reporter assays showed that overexpression of miR-204 significantly decreased the relative luciferase activity of Slug-3'UTR in HEK293T and HuH28 cells, but had no effect on the mutant of Slug-3'UTR (Fig. 4B). Moreover, qRT-PCR, western blotting and immunofluorescence showed that miR-204 overexpression substantially decreased the expression of Slug in HuH28 cells, and that miR204 depletion increased Slug expression in HuCCT1 cells (Fig. 4C and D). Taken together, these results suggest that Slug is a direct of miR-204. 


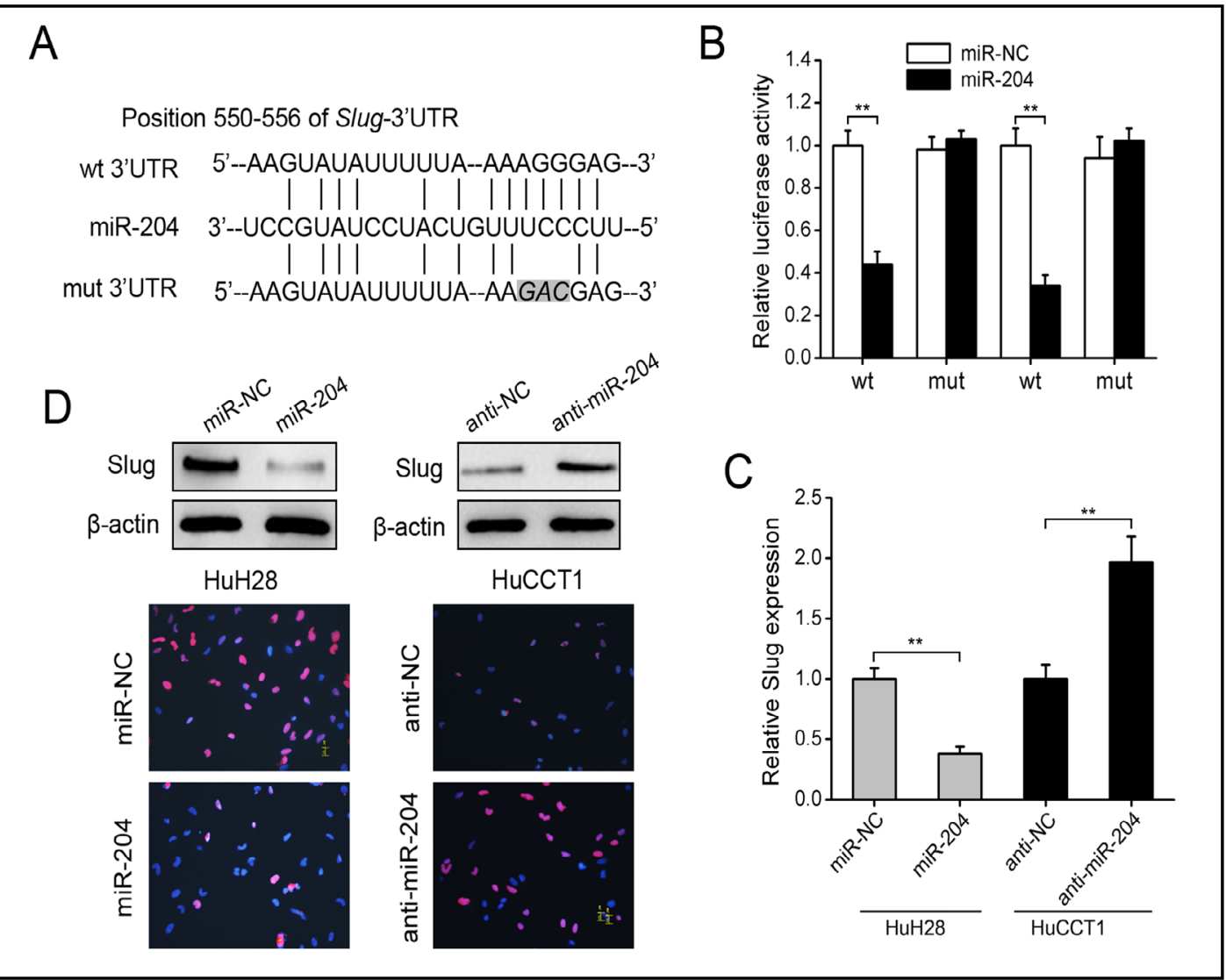

Fig. 4. Slug is a direct target of miR-204. (A) The putative binding sequences of miR-204 in the 3'UTR of Slug gene. Mutation was generated as indicated. Either wild-type (WT) or mutant (Mut) Slug 3'UTR was cloned into the psiCHECK2 vector. (B) Relative luciferase activity in HEK293T and HuH28 cells co-transfected with miR-204 and a luciferase reporter containing the Slug 3'UTR (WT) or a mutant (Mut). (C) The expression of Slug mRNA in HuH28 and HuCCT1 cells was mesured by qRT-PCR. $\beta$-actin was used as an internal control. (D) Left: western blotting and immunofluorescence analyses of the endogenous Slug protein in miR-204 overexpressing HuH28 cells or negative control. Right: western blotting and immunofluorescence analyses of the endogenous Slug protein in HuCCT1 cells transfected with anti-miR-204 or anti-NC. ${ }^{* *} P<0.01$.

\section{Re-expression of Slug partially rescues miR-204-induced suppression of EMT, cell migration and invasion}

To further determine whether miR-204 exerts its function through repression of Slug, we performed gain-of-function analyses by overexpressing Slug in miR-204-overexpressing HuH28 cells. As expected, overexpression of Slug partially rescued the morphological change caused by overexpression of miR-204 in HuH28 cells (Fig. 5A). The upregulation of vimentin and the the downregulation of E-cadherin were also observed (Fig. 5B). Consistent with these results, the restoration of Slug expression antagonized the inhibitory effect of miR-204 on cell migration and invasion (Fig. 5C). Taken together, these results suggest that Slug is a functional target of miR-204.

miR-204 and Slug are inversely expressed in ICC specimens

To further investigate whether miR-204-induced modulation of Slug is of clinical relevance, we assessed the expression levels of Slug in clinical ICC tissues. Both qRT-PCR and immunohistochemistry showed that Slug expression was increased in ICC tissues with lymph node metastases compared with the lymph-node-negative primary ICC tissues (Fig. 
A
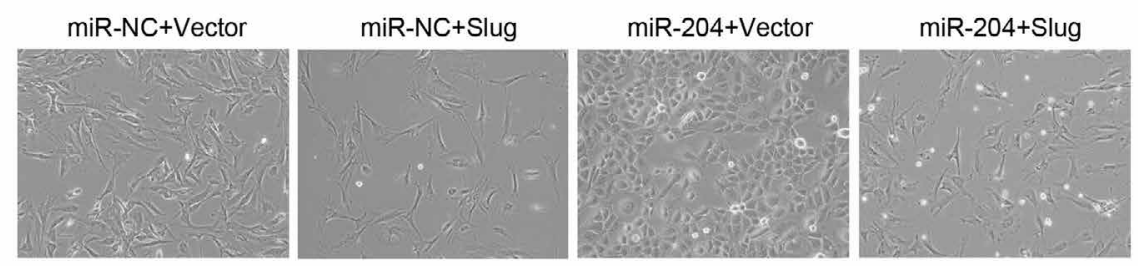

B

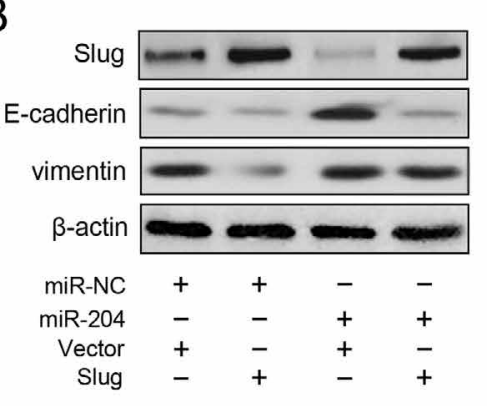

C

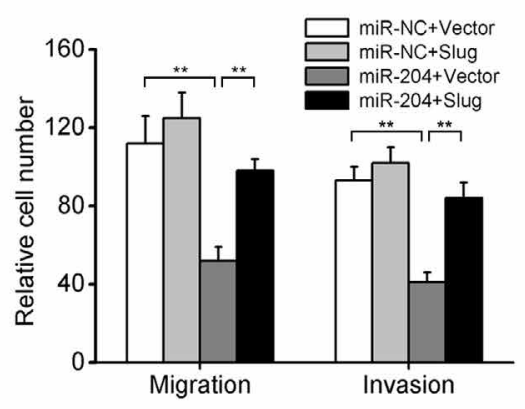

Fig. 5. Re-expression of Slug partially rescues miR-204-induced suppression of EMT, cell migration and invasion in HuH28 cells. (A) Morphology of miR-204 overexpressing HuH28 cells infected with Slug lentivirus or control. (B) western blotting analysis of miR-204 overexpressing HuH28 cells infected with Slug lentivirus or control. (C) Transwell migration and invasion assays of miR-204 overexpressing HuH28 cells infected with Slug lentivirus or control. ${ }^{* *} P<0.01$.

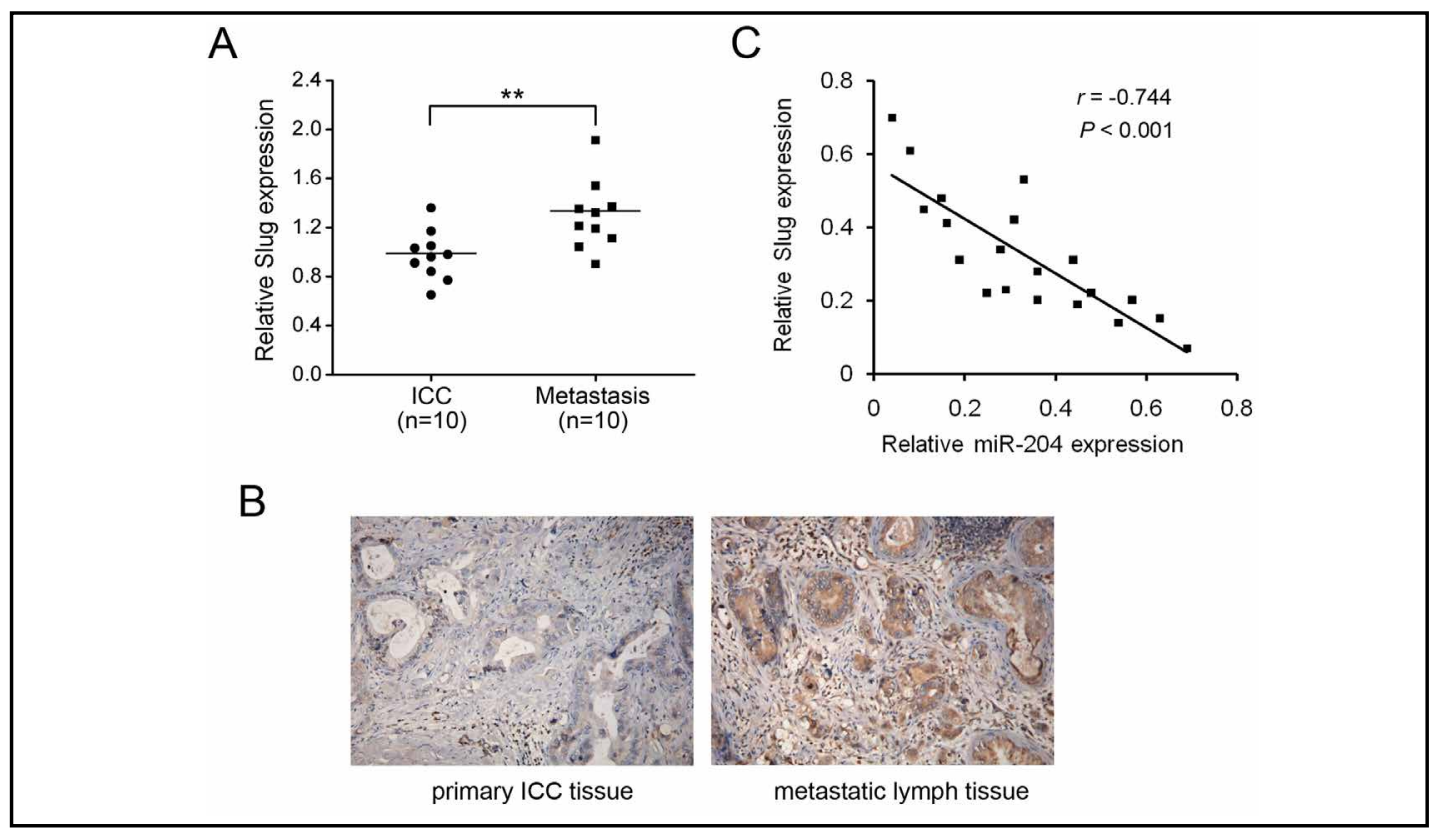

Fig. 6. miR-204 and Slug are inversely expressed in ICC specimens. (A) Relative expression of Slug mRNA in primary ICC tissues and lymph node metastatic tissues. (B) Expression of Slug in primary ICC tissues and lymph node metastatic tissues by immunohistochemistry (IHC). (C) Spearman's correlation analysis shows a significant inverse correlation between miR-204 expression level and Slug mRNA level in ICC tissues. ${ }^{* *} P<0.01$.

6A and B) Furthermore, statistical analysis revealed that miR-204 levels were inversely correlated with the expression of Slug mRNA (Fig. 6C). 


\section{Discussion}

In this study, we showed that miR-204 was frequently downregulated in human ICC, and the low-level expression of miR-204 was significantly associated with a more aggressive tumour phenotype. In functional studies, overexpression of miR-204 dramatically suppressed ICC cell migration and invasion. We identified Slug as a direct target of miR-204, and its downregulation by miR-204 in HuH28 cells reversed EMT, as shown by the increased expression of the epithelial marker E-cadherin and decreased expression of the mesenchymal marker vimentin. These findings suggest that miR-204 may play important roles in the invasive and/or metastatic potential of ICC.

Downregulation of miR-204 is a frequent event in several cancers [20-25]. Recently, mounting evidence indicate that miR-204 play crucial roles in tumor invasion and metastasis. Ying et al. showed that miR-204 simultaneously suppresses self-renewal, stem cell-associated phenotype, and migration of glioma cells. miR-204 also inhibits cell invasion and metastasis of breast cancer and ovarian cancer cells [24]. In this study, we also found that miR-204 was frequently downregulated in ICC tissues compared to their matched normal tissues, and the downregulated miR-204 was significantly associated with ICC metastasis. We subsequently confirmed that miR-204 overexpression significantly suppressed ICC cell migration and invasion, suggesting that miR-204 acts as a metastasis suppressor in ICC.

A proposed critical step in the progression of primary tumors toward invasion and metastasis is attributed to the process known as epithelial-to-mesenchymal transition (EMT). The well-established hallmark of EMT is the loss of E-cadherin, which is a prerequisite for epithelial tumor cell invasion [27]. In this study, we observed that overexpression of miR-204 in HuH28 cells induced morphological changes from an elongated, fibroblast-like phenotype to an epithelial cobblestone-like phenotype. Meanwhile, miR-204 overexpression significantly enhanced E-cadherin expression but decreased the expression of vimentin. Conversely, inhibition of miR-204 in HuCCT1 cells greatly increased E-cadherin expression. Furthermore, we found that miR-204 overexpression significantly reduced Slug expression, suggesting that Slug may play an important role in the miR-204-induced cell effects.

Slug, a member of the snail family of transcription factors, plays important roles in the regulation of EMT by suppressing the epithelial marker E-cadherin [28, 29]. Multiple lines of evidence suggest that upregulation of Slug contributes to invasive and metastatic behavior in several cancers [30-33]. Furthermore, Zhang et al. showed that the expression of slug protein was frequently detected in ICC tissues; however, slug was not expressed in normal intrahepatic bile ducts or liver parenchyma. They also found that Slug was closely correlated with lymph node invasion [34]. In this study, we confirmed that Slug was a novel, direct and functional target of miR-204. This conclusion is supported by the following evidence: miR-204 overexpression decreased the luciferase reporter activity of wild-type 3'UTR but not mutant 3'UTR of Slug; Up-regulation of miR-204 significantly suppressed Slug mRNA and protein expression in ICC cells, whereas down-regulation of miR-204 increased Slug expression; the expression of miR-204 correlated inversely with the expression of Slug in human ICC tissues; overexpression of Slug in miR-204 overexpressing HuH28 cells rescued the suppressive effect of miR-204 on EMT, cell migration and invasion. These results demonstrate that the frequently downregulated miR-204 in ICC lead to the increased expression of Slug and in turn contribute to ICC metastasis and progression.

In conclusion, our results show that miR-204 is significantly downregulated in ICC tissues and cell lines. Overexpression of miR-204 negatively regulates EMT and suppresses cell migration and invasion. Furthermore, Slug is a direct and functional target of miR-204, and miR-204 exerts its function through downregulating Slug expression. Our data suggest that these molecules might represent potential targets for future treatment of human ICC. 


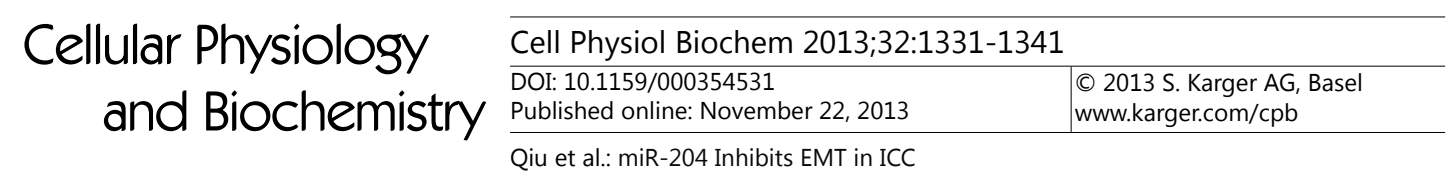

\section{Acknowledgements}

This work was supported by the Shanghai Health Bureau of China (No. 20124165).

\section{References}

1 Shaib Y, El-Serag HB: The epidemiology of cholangiocarcinoma. Semin Liver Dis 2004;24:115-125.

- Taylor-Robinson SD, Toledano MB, Arora S, Keegan TJ, Hargreaves S, Beck A, Khan SA, Elliott P, Thomas HC: Increase in mortality rates from intrahepatic cholangiocarcinoma in England and Wales 1968-1998. Gut 2001;48:816-820.

3 Nguyen DX, Bos PD, Massague J: Metastasis: from dissemination to organspecific colonization. Nat Rev Cancer 2009;9:274-365.

4 Wang B, Lindley LE, Fernandez-Vega V, Rieger ME, Sims AH, Briegel KJ: The T box transcription factor TBX2 promotes epithelial-mesenchymal transition and invasion of normal and malignant breast epithelial cells. PLoS One 2012;7:e41355.

5 Taube JH, Herschkowitz JI, Komurov K, Zhou AY, Gupta S, Yang J, Hartwell K, Onder TT, Gupta PB, Evans KW, Hollier BG, Ram PT, Lander ES, Rosen JM, Weinberg RA, Mani SA: Core epithelial-to-mesenchymal transition interactome gene-expression signature is associated with claudin-low and metaplastic breast cancer subtypes. Proc Natl Acad Sci USA 2010;107:15449-15454.

6 Thiery JP: Epithelial-mesenchymal transitions in tumour progression. Nat Rev Cancer 2002;2:442-454.

7 Thompson EW, Williams ED: EMT and MET in carcinoma-clinical observations, regulatory pathways and new models. Clin Exp Metastasis 2008;25:591-592.

8 Peinado H, Marin F, Cubillo E, Stark HJ, Fusenig N, Nieto MA, Cano A: Snail and E47 repressors of E-cadherin induce distinct invasive and angiogenic properties in vivo. J Cell Sci 2004;117:2827e39.

-9 Polyak K, Weinberg RA: Transitions between epithelial and mesenchymal states: acquisition of malignant and stem cell traits. Nat Rev Cancer 2009;9:265-273.

10 Plasterk RH: Micro RNAs in animal development. Cell 2006;124:877-881.

11 Cho WC: MicroRNAs in cancer-from research to therapy. Biochem Biophys Acta 2010;1805:209-217.

12 Kong KL, Kwong DL, Chan TH, Law SY, Chen L, Li Y, Qin YR, Guan XY: MicroRNA-375 inhibits tumour growth and metastasis in oesophageal squamous cell carcinoma through repressing insulin-like growth factor 1 receptor. Gut 2012;61:33-42.

13 Rosenfeld N, Aharonov R, Meiri E, Rosenwald S, Spector Y, Zepeniuk M, Benjamin H, Shabes N, Tabak S, Levy A, Lebanony D, Goren Y, Silberschein E, Targan N, Ben-Ari A, Gilad S, Sion-Vardy N, Tobar A, Feinmesser M, Kharenko O, Nativ O, Nass D, Perelman M, Yosepovich A, Shalmon B, Polak-Charcon S, Fridman E, Avniel A, Bentwich I, Bentwich Z, Cohen D, Chajut A, Barshack I: MicroRNAs accurately identify cancer tissue origin. Nat Biotechnol 2008;26:462e9.

14 Karakatsanis A, Papaconstantinou I, Gazouli M, Lyberopoulou A, Polymeneas G, Voros D: Expression of microRNAs, miR-21, miR-31, miR-122, miR-145, miR-146a, miR-200c, miR-221, miR-222, and miR-223 in patients with hepatocellular carcinoma or intrahepatic cholangiocarcinoma and its prognostic significance. Mol Carcinog 2013;52:297-303.

$>15$ Zeng B, Li Z, Chen R, Guo N, Zhou J, Zhou Q, Lin Q, Cheng D, Liao Q, Zheng L, Gong Y: Epigenetic regulation of miR-124 by hepatitis $C$ virus core protein promotes migration and invasion of intrahepatic cholangiocarcinoma cells by targeting SMYD3. FEBS Lett 2012;586:3271-3278.

16 Li B, Han Q Zhu Y, Yu Y, Wang J, Jiang X: Down-regulation of miR-214 contributes to intrahepatic cholangiocarcinoma metastasis by targeting Twist. FEBS J 2012;279:2393-2398.

17 Gregory PA, Bert AG, Paterson EL, Barry SC, Tsykin A, Farshid G, Vadas MA, Khew-Goodall Y, Goodall GJ: The miR-200 family and miR-205 regulate epithelial to mesenchymal transition by targeting ZEB1 and SIP1. Nat Cell Biol 2008;10:593-601.

18 Oishi N, Kumar MR, Roessler S, Ji J, Forgues M, Budhu A, Zhao X, Andersen JB, Ye QH, Jia HL, Qin LX, Yamashita T, Woo HG, Kim YJ, Kaneko S, Tang ZY, Thorgeirsson SS, Wang XW: Transcriptomic profiling reveals hepatic stem-like gene signatures and interplay of miR-200c and epithelial-mesenchymal transition in intrahepatic cholangiocarcinoma. Hepatology 2012;56:1792-1803. 


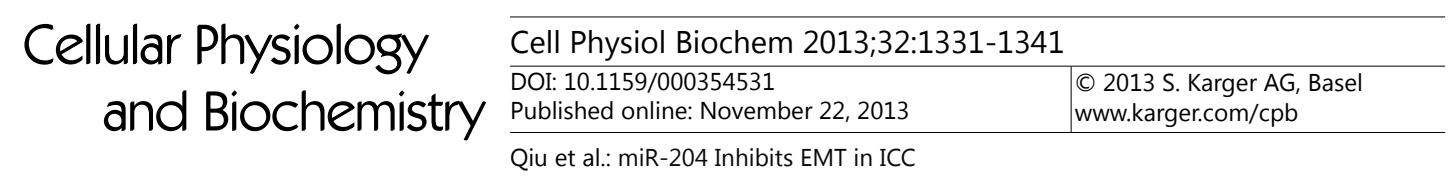

19 Chen L, Yan HX, Yang W, Hu L, Yu LX, Liu Q, Li L, Huang DD, Ding J, Shen F, Zhou WP, Wu MC, Wang HY: The role of microRNA expression pattern in human intrahepatic cholangiocarcinoma. J Hepatol 2009;50:358369.

20 Chung TK, Lau TS, Cheung TH, Yim SF, Lo KW, Siu NS, Chan LK, Yu MY, Kwong J, Doran G, Barroilhet LM, Ng AS, Wong RR, Wang VW, Mok SC, Smith DI, Berkowitz RS, Wong YF: Dysregulation of microRNA-204 mediates migration and invasion of endometrial cancer by regulating FOXC1. Int J Cancer 2012;130:10361045.

21 Gong M, Ma J, Li M, Zhou M, Hock JM, Yu X: MicroRNA-204 critically regulates carcinogenesis in malignant peripheral nerve sheath tumors. Neuro Oncol 2012;14:1007-1017.

-22 Sacconi A, Biagioni F, Canu V, Mori F, Di Benedetto A, Lorenzon L, Ercolani C, Di Agostino S, Cambria AM, Germoni S, Grasso G, Blandino R, Panebianco V, Ziparo V, Federici O, Muti P, Strano S, Carboni F, Mottolese M, Diodoro M, Pescarmona E, Garofalo A, Blandino G: miR-204 targets Bcl-2 expression and enhances responsiveness of gastric cancer. Cell Death Dis 2012;3:e423.

23 Zhang L, Wang X, Chen P: MiR-204 down regulates SIRT1 and reverts SIRT1-induced epithelialmesenchymal transition, anoikis resistance and invasion in gastric cancer cells. BMC Cancer 2013;13:290.

24 Ying Z, Li Y, Wu J, Zhu X, Yang Y, Tian H, Li W, Hu B, Cheng SY, Li M: Loss of miR-204 expression enhances glioma migration and stem cell-like phenotype. Cancer Res 2013;73:990-999.

25 Zeng L, Yu J, Huang T, Jia H, Dong Q He F, Yuan W, Qin L, Li Y, Xie L: Differential combinatorial regulatory network analysis related to venous metastasis of hepatocellular carcinoma. BMC Genomics 2012;13:S14.

26 Xiong S, Zheng Y, Jiang P, Liu R, Liu X, Chu Y: MicroRNA-7 inhibits the growth of human non-small cell lung cancer A549 cells through targeting BCL-2. Int J Biol Sci 2011;7:805-814.

27 Kong X, Li G, Yuan Y, He Y, Wu X, Zhang W, Wu Z, Chen T, Wu W, Lobie PE, Zhu T: MicroRNA-7 inhibits epithelial-to-mesenchymal transition and metastasis of breast cancer cells via targeting FAK expression. PLoS One 2012;7:e41523.

28 Nieto MA: The snail superfamily of zinc-finger transcription factors. Nat Rev Mol Cell Biol 2002;3:155-166.

29 Hajra KM, Chen DY, Fearon ER: The SLUG zinc-finger protein represses E-cadherin in breast cancer. Cancer Res 2002;62:1613-1618.

30 Shih JY, Tsai MF, Chang TH, Chang YL, Yuan A, Yu CJ, Lin SB, Liou GY, Lee ML, Chen JJ, Hong TM, Yang SC, Su JL, Lee YC, Yang PC: Transcription repressor slug promotes carcinoma invasion and predicts outcome of patients with lung adenocarcinoma. Clin Cancer Res 2005;11:8070-8078.

31 Chen M, Chen LM, Chai KX: Androgen regulation of prostasin gene expression is mediated by sterolregulatory element-binding proteins and SLUG. Prostate 2006;66:911-920.

- 32 Yang HW, Menon LG, Black PM, Carroll RS, Johnson MD: SNAI2/Slug promotes growth and invasion in human gliomas. BMC Cancer 2010;10:301.

33 Zhang K, Chen D, Jiao X, Zhang S, Liu X, Cao J, Wu L, Wang D: Slug enhances invasion ability of pancreatic cancer cells through upregulation of matrix metalloproteinase- 9 and actin cytoskeleton remodeling. Lab Invest 2011;91:426-438.

-34 Zhang KJ, Zhang BY, Zhang KP, Tang LM, Liu SS, Zhu DM, Zhang DL: Clinicopathologic significance of slug expression in human intrahepatic cholangiocarcinoma. World J Gastroenterol 2010;16:2554-2557. 